\title{
Identification of a one-bit lowpass sigma-delta modulator using BIMBO
}

\author{
E. Colinet, CEA-LETI, DCIS/SCME/LMEA, France \\ J. Juillard, SUPELEC, SSE, France
}

\begin{abstract}
We show how the BIMBO identification method can be used for the online identification of the digital filter in a sigma-delta modulator's loop. This parameter estimation method only requires the knowledge of the bit stream at the modulator output. This approach does not rely on an approximation of the comparator (such as additive white noise or describing function approximation). Moreover, it requires no amplitude measurement, as opposed to most other methods, and, thus, it involves no additional electronics and it is therefore very economical to implement it practically. The method is described from a theoretical point of view and an experimental validation is given.
\end{abstract}

Keywords - sigma-delta conversion, parameter estimation, self-calibration.

\section{Introduction}

Sigma-delta A/D converters are nowadays widely used in numerous applications, such as audio applications [12]. They also have received a lot of attention in the field of MEMS [3-4] and of integrated electronics because they do not require delicate or surface-consuming analog components, as opposed to traditional A/D converters. Sigma-delta A/D converters consist of two parts (Fig. 1): a sigma-delta modulator and a (digital) filtering decimation stage. The modulator outputs a one-bit signal which is equivalent to the input signal in a limited bandwidth. It is designed according to the following two principles:

- the input signal is sampled at a rate much larger than the Nyquist rate (oversampling),

- the quantization noise introduced by the comparator is rejected out of the band of interest (noise shaping).

Considering the classical linear approximation which consists in replacing the comparator by an additive white noise $e$, one can write:

$s=S T F \cdot e+N T F \cdot b$,

where $S T F=\frac{H\left(z^{-1}\right)}{1+H\left(z^{-1}\right)}$ and $N T F=\frac{1}{1+H\left(z^{-1}\right)}$.

Equation (1) shows that the modulator output $s$ can not be used as is: it must be processed through several filtering and decimation stages, which reduce the signal rate and filter out the quantization noise. Moreover, since the $S T F$ is usually different from unity, a deconvolution process must be employed in order to find out an exact representation of signal $e$, meaning that the transfer function $H$ has to be known. This becomes problematic in the field of integrated electronics, because several dispersion sources exist (e.g. due to the fabrication process or to environmental variations), which may result in an imperfect knowledge of $H$ and, thus, decrease the overall performance of the converter. Ideally, a self-calibration function should then be added to the A/D converter so that $H$ can be monitored, its parameters estimated and the coefficients of the digital signal processing part of the converter adjusted accordingly.

Most existing identification schemes rely on a measurement of the amplitude of a system's output: this is also true of sigma-delta specific methods such as OBT [5]. This implies that the system's output is usually quantized with a high resolution so that the quantization noise is as low as possible. It is very difficult to use this approach in the context of sigma-delta converters: the output of the converter (after the filtering and decimation stages) has a high resolution but it holds very little information about $H$. On the other hand, the output of the comparator does contain information about $H$, but with a very low resolution.

In this paper we describe an original method for the estimation of the parameters of one-bit sigma-delta modulators, that does not rely on an amplitude measurement. It is based on the BIMBO (Basic Identification Method using Binary Observations) method [6], which is a generic identification process for systems with binary outputs. The rest of this paper is organized as follows: first, the BIMBO method is described from a theoretical point of view. Then, we show how it can be applied to sigma-delta converters and, more generally, to sigmadelta-based systems and discuss different approaches: closed or open loop, with or without prior knowledge of the system's input. Some experimental results are given in the case of a lowpass sigma-delta A/D converter.

\section{BIMBO}

The BIMBO method is a more powerful, less limiting version of the MLCF and MCLC identification methods [6-9] for relay feedback systems. The common point between the three methods is that they do not rely on an 
approximation (such as additive white noise or describing function approximation) of the comparator, as opposed to other methods [5,10,11]. However, BIMBO has none of the drawbacks of the other two methods:

- it does not rely on a slow self-sustained oscillation of a closed-loop system, which can be difficult to obtain in the context of sigma-delta converters,

- the only components it requires are a one-bit ADC and a one-bit DAC.

The principle of BIMBO is the following (see Fig. 2 for notations):

- $\quad$ generate a spectrally rich signal $u_{k}$, such as a white noise. This signal can be a one-bit signal so that the DAC can be implemented with ease.

- $\quad$ measure the system's output $y_{k}$ using a one-bit ADC, i.e. measure $s_{k}=S\left(y_{k}\right)$

- using a discrete parametric model of the system, compute the estimated time response $\hat{y}_{k}$ and $\hat{s}_{k}=S\left(\hat{y}_{k}\right)$

- $\quad$ adjust the parametric model of the discrete system so that a correct estimation $\hat{y}_{k}$ is produced.

An optimization algorithm is necessary for this last step. Letting $\theta$ be the unknown system parameters, the criterion that must be minimized is:

$J(\theta)=\sum_{k=1 \ldots N}\left(s_{k}-S\left(\hat{y}_{k}\right)\right)^{2} \hat{y}_{k}^{2}$,

where $N$ is the number of samples and $S($.$) is the comparator's input-output relationship - usually, S($.$) is the$ sign function.

It is simple to verify that this criterion is smooth and that it is convex when $\theta$ is chosen to represent the coefficients of the unknown system's impulse response. In this case, a simple gradient algorithm is enough to identify $\theta$. When the unknown system has an infinite impulse response, it is more economical to take for unknowns the coefficients of an ARX model of the system. This usually leads to a non-convex criterion, which can be optimized using, for example, a Hooke and Jeeves algorithm or simulated annealing.

For a finite number of samples, there usually exists one volume of $\theta$-space for which criterion (2) is zero (and, thus, is minimal). It has been shown that, as the number of samples goes to infinity, this volume reduces to a point.

\section{Application of BIMBO to a sigma-delta modulator}

\section{a) Principle}

BIMBO can be applied online to identify the transfer function $H$ of a lowpass sigma-delta modulator using only the bit stream at the comparator output. Suppose there is an unknown but constant signal $e$ at the modulator input - which is the normal operating regime of a lowpass sigma-delta $\mathrm{A} / \mathrm{D}$ converter. One may, as a first estimate of $e$, use the averaged output:

$\hat{e}=\frac{1}{N} \sum_{k=1}^{N} s_{k}$.

Subtracting the known (measured) signal $s_{k}=\operatorname{sign}\left(y_{k}\right)$ from (3) yields an estimate of the unknown transfer function's input:

$\hat{u}_{k}=\hat{e}_{k}-s_{k}$.

BIMBO can then be used in a first iteration step to identify a set of parameters of $H$ using the approach described in the previous section. This process must then be iterated: the set of parameters obtained at the $(n-1)^{\text {th }}$ step is used to refine the estimation of input $e$; this new estimation is used to update $\hat{u}_{k}$ and BIMBO yields an $n^{\text {th }}$ set of parameters for $H$, until convergence is reached. In case the input signal $e_{k}$ is known or imposed (in which case it is more efficient to use a spectrally rich signal), there is no need for this iterative process and the parameters of $H$ are obtained directly using:

$u_{k}=e_{k}-s_{k} \cdot(5)$

Finally, it is also possible to use open-loop BIMBO, that is to open the sigma-delta feedback loop and generate an input signal $e_{k}$, so that:

$u_{k}=e_{k}$.

\section{b) Experimental results}

The online approach described in the previous subsection has been experimentally validated in the case of lowpass sigma-delta modulator. In this case, the theoretical transfer function is designed to be: 


$$
H_{\text {theoretical }}=\frac{5.891 e^{-3} z^{-1}-4.124 e-3 z^{-2}}{1-1.889 z^{-1}+0.8911 z^{-2}}=5.891 e^{-3} \frac{(z-0.7001)}{(z-0.9807)(z-0.9087)}
$$

This filter was implemented using switched capacitances. The complete circuit was fabricated using a $0.35 \mu \mathrm{m}$ CMOS technology (Fig. 4). Testing of the circuit revealed that the performances were not as expected. Using the online method described above, an identification of $H$ is done, based on the ARX structure of (8). 10000 sample points are used. The input is a constant but its value is not precisely known. After a few iterations, the algorithm yields:

$$
H_{\mathrm{BIMBO}}=G \frac{(z+0.1152)}{(z-0.9658)(z-0.7537)}(9)
$$

Note that gain $G$ cannot be identified using BIMBO. However, this coefficient has only a very moderate impact on the behaviour of the sigma-delta modulator (it only comes into play in the case of an imperfection of the comparator, such as a dead zone). Comparing (8) and (9) shows that there is an important dispersion between parameters of $H_{\text {theoretical }}$ and $H_{\mathrm{BIMBO}}$. This dispersion comes from imperfections in the electronic design. In order to verify which model represents the real circuit better, the theoretical performances of the modulator are calculated using Matlab/Simulink, in the case $H=H_{\text {theoretical }}$ and in the case $H=H_{\mathrm{BIMBO}}$. The resulting outupt spectra are then compared to the one that is observed. Fig. 5 shows clearly that $H_{\mathrm{BIMBO}}$ is more representative of the real system than $H_{\text {theoretical }}$.

\section{Conclusion}

We have shown how BIMBO can be used for the online identification of the digital filter in a sigma-delta modulator's loop. This parameter estimation method only requires the knowledge of the bit stream at the modulator output. Thus, as opposed to other methods, it requires no additional electronics and it is therefore very economical to implement it practically. BIMBO has demonstrated great robustness towards model's imperfections (noise, non-linearity, etc.). It has been validated in several cases such as a lowpass sigma-delta A/D converter (presented here), a lowpass sigma-delta micro-accelerometer or a bandpass sigma-delta A/D converter and more exotic systems with binary outputs, such as a "Game of Life" cellular automaton. Our current research is focussed on the implementation of BIMBO in a real-time application so that the decimation filter can be adjusted to the parameters of the identified filter.

\section{References}

[1] J. C. Candy and G. C. Temes, Oversampling Delta-Sigma Data Converters, IEEE, New York, 1992.

[2] T. Ritoniemi, et al., A stereo audio sigma-delta A/D-converter, IEEE Journal of Solid-State Circuits, vol. 29, 1514-1523, 1994.

[3] X. Jiang, et al., A monolithic surface micromachined Z-axis gyroscope with digital output, Symposium on VLSI Circuits, 2000, pp. 16-19.

[4] M. Kraft, Closed loop digital accelerometer employing oversampling conversion, $\mathrm{Ph}$. D. Thesis, University of Coventry, U.K., 1997.

[5] G. Huertas, et al., Oscillation-based test in oversampled SD modulators, Microelectronics Journal, vol. 33, pp. 799-806, 2002.

[6] E. Colinet, Nouvelles architectures et méthodes de conception de microsystèmes sigma-delta et de microsystèmes résonants, Ph. D. Thesis, University Paris-Sud Orsay, France, 2005.

[7] E. Colinet, et al., Auto-calibrage d'un micro-accéléromètre par une mesure fréquentielle, $3^{\mathrm{e}}$ Colloque Interdisciplinaire en Instrumentation, 2004, vol. 1, pp. 355-362.

[8] E. Colinet, et al., Digital self-calibration method for MEMS sensors, IEEE Transactions on Instrumentation and Measurement, vol. 54, pp. 1438-1443, 2005

[9] E. Colinet, et al., Application of MCLC method for estimating the parameters of MEMS sensors, NSTI Nanotechnology Conference and Trade Show, 2005, pp. 335-33.

[10] D. De Venuto, Testing high resolution $\Sigma \Delta A D C$ 's by using the quantizer input as test access, Microelectronics Journal, vol. 36, pp. 810-19, 2005.

[11] K. Voda, I..D. Landau, The autocalibration of PI controllers based on two frequency measurements, International Journal of Adaptive Control and Signal Processing, vol. 9, pp. 395-421, 1995. 


\section{Figures}

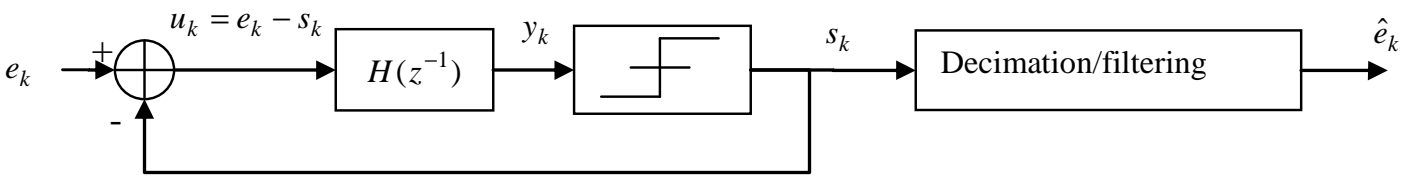

Fig.1 - Basic structure of a sigma-delta A/D converter.

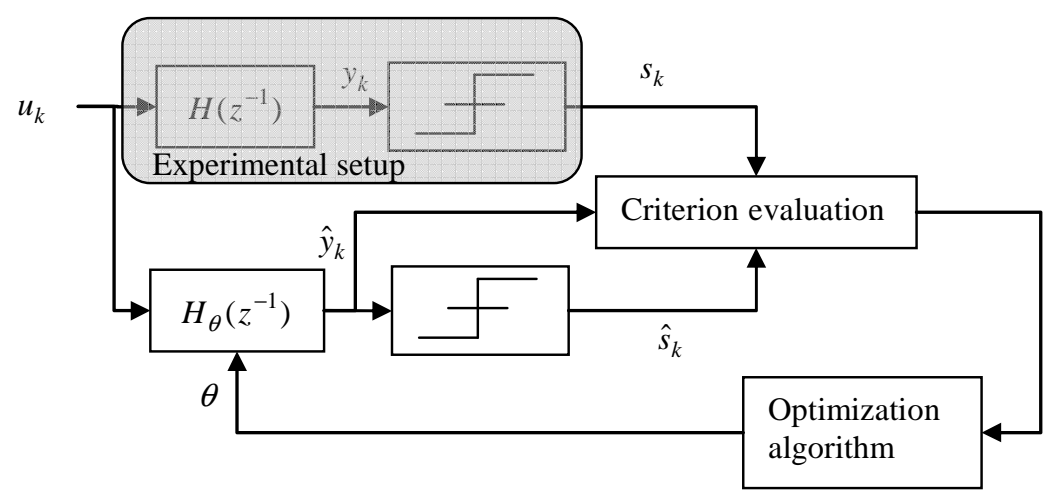

Fig. 2 - Block diagram of the BIMBO method. A parametric model of the real system is built so as to maximize the similarity between $s_{k}$ and $\hat{s}_{k}$. A smooth criterion is given by (2).

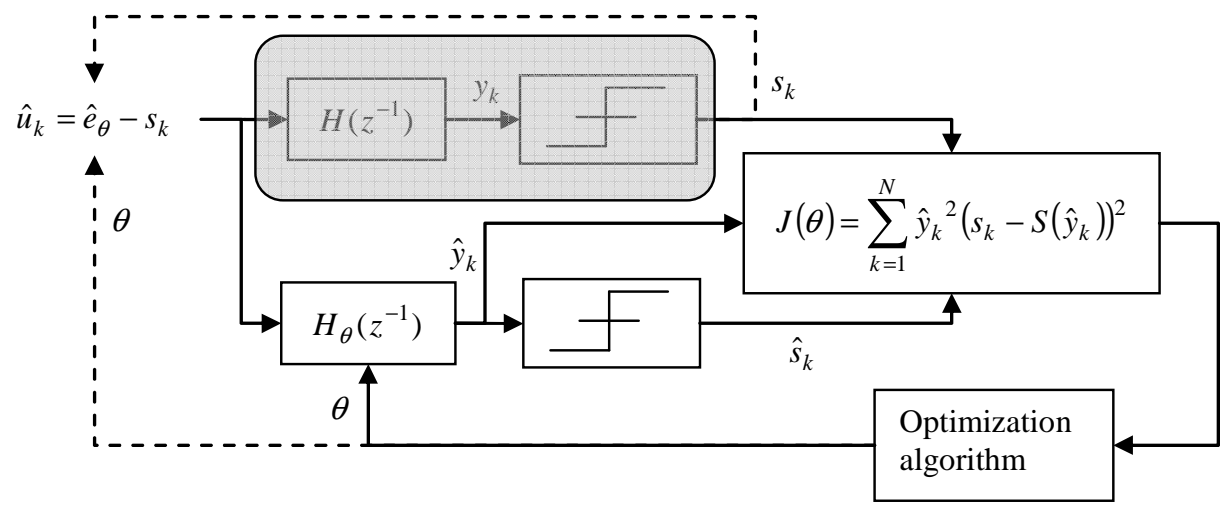

Fig. 3 - Block diagram of the BIMBO method for the online identification of a (lowpass) sigma-delta modulator. The input of the parametric model is estimated using the current value of $\theta$. 


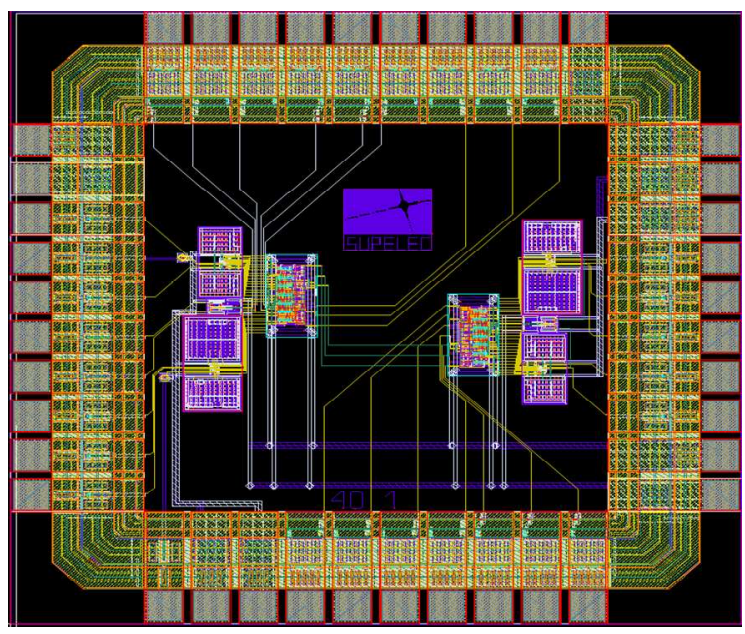

Fig. 4 - Circuit layout of the lowpass sigma-delta modulator.

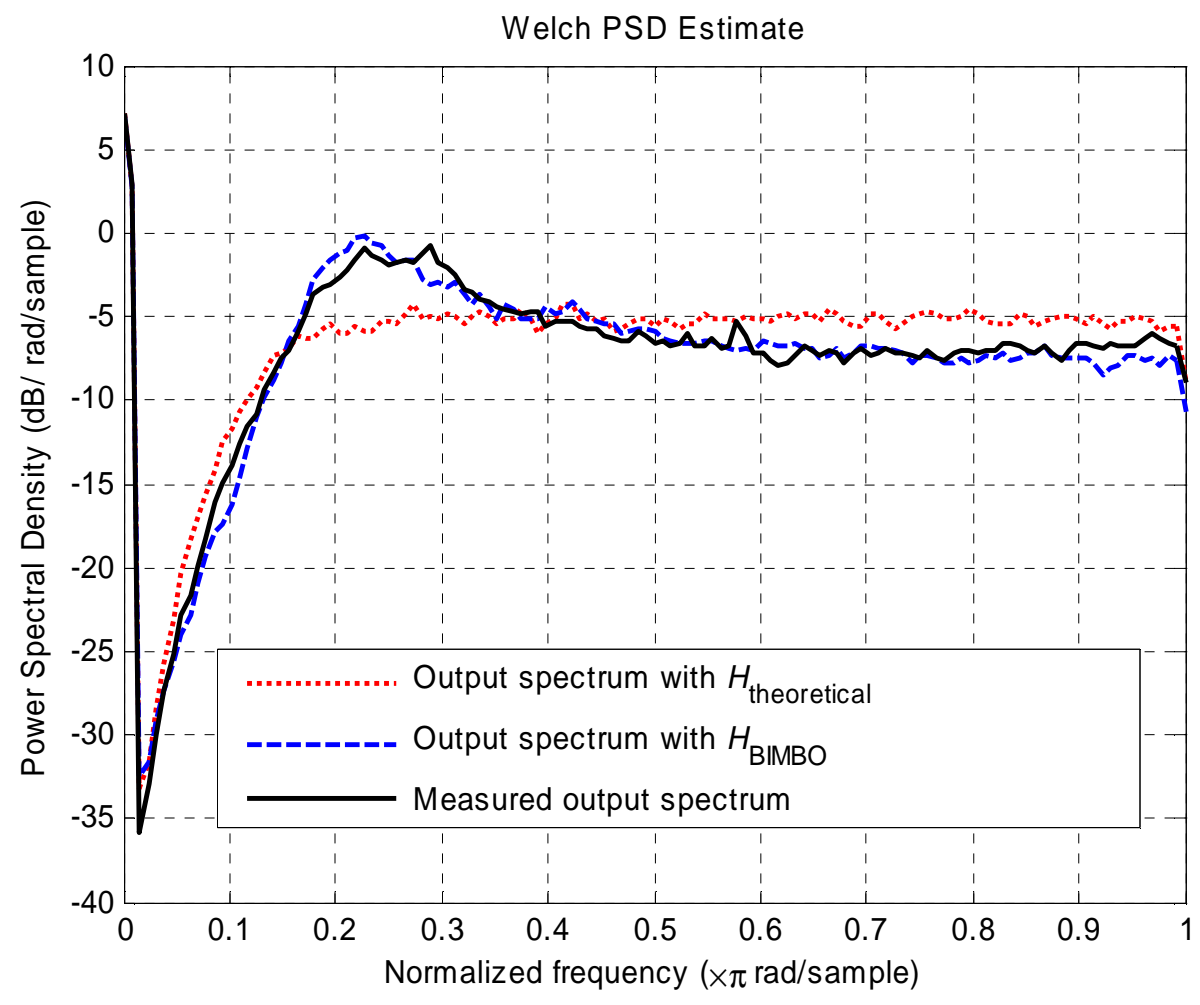

Fig. 5 - Comparison between the experimental and simulated sigma-delta output spectra. The output spectrum obtained $H_{\mathrm{BIMBO}}$ fits the experimental spectrum better than the one obtained with $H_{\text {theoretical }}$. 